\title{
La RFG fait son cinéma
}

W $\begin{aligned} & \text { ous sommes en } 2018 \text {, et qu'ils paraissent loin, déjà, les } \\ & \text { temps pas si anciens... }\end{aligned}$ 2012, lorsque la RFG était classée rang 4 Fnege et rang 4 CNRS. Doucement mais sûrement, la revue était poussée vers la sortie et donc la pré-retraite scientifique. Une revue de langue française, sans ambition internationale donc, à quoi cela pouvait-il encore servir? Sonnée, K.O., prête à être "descendue des plateaux de sono académiques » par des revues d'associations plus belles et plus fortes qu'elle, la RFG n'avait plus qu'à méditer les chansons d'Alain Souchon. Et se dire que décidément, dans les arts, les affaires, comme les sciences, il arrive toujours un moment où on a fait son temps. Sauf à avoir produit des chefs-d'œuvres.

2013, in extremis pour ne pas dire - déjà ! - de haute lutte, un petit sursis a été accordé : la remontée en rang 3 dans la liste FNEGE élaborée par le collège des associations académiques... Parce que quand même, subsistait ce doute : elle avait beau être francophone, elle avait beau être généraliste, la RFG portait beau sous Google Scholar: $\mathrm{n}^{\circ} 1$ des revues francophones à l'époque, toutes catégories confondues. Un sursis utile, qui permettait au moins de réamorcer la pompe des soumissions et donc de stimuler aussi les évaluateurs.

2014, la RFG a pris l'initiative de proposer un partenariat académique aux équipes de Xerfi Canal Productions TV. Quatre ans plus tard, plus de 200 interviews de recherche et des 
dizaines d'heures sont disponibles et mobilisées partout, en particulier dans les salles de cours. Les mondes professionnel et académique ont compris l'intérêt réciproque qu'ils pouvaient avoir non plus à s'ignorer mais à se parler et à mobiliser leurs compétences respectives. Une belle victoire, pour les deux partenaires.

2015, c'était l'année du lancement du site The Conversation France. Celui-ci a légitimement gagné ses lettres de noblesse et la RFG est heureuse et fière d'avoir pu apporter sa modeste contribution à ce succès. La revue, ses auteurs et leurs institutions ont bénéficié d'effets d'exposition inconnus jusqu'alors. Couplés aux effets des vidéos RFG-XERFI, la valse est devenue à trois temps. Une réussite partenariale aussi exemplaire qu'inédite.

C'est donc logiquement qu'en 2016 (mais après deux tours de vote, quand même...), la RFG a gravé sa percée dans le marbre de la liste des classement des revues Fnege : rang 2 ! Le CNRS n'a pu que suivre et reconnaître cette «verrue » qui décrédibilisait son propre classement historique : la RFG a été remontée en rang 3 CNRS. Il faut dire qu'avec plus de 400 soumissions par an, une base de plus de 900 évaluateurs, un taux de sélection des articles de $15 \%$, il aurait été difficile de faire autrement.

Puisque pour filer les célèbres travaux de Danny Miller (membre très actif du Conseil de rédaction de la RFG depuis 2013) et de son paradoxe d'Icare, le succès conduit mécaniquement à l'échec, il était donc temps de se réinventer, déjà, dès 2017. Cette idée s'est donc imposée : la RFG pouvait et devait organiser des manifesta- tions, puisque ses huit numéros par an appelaient une valorisation aussi sous la forme du spectacle vivant, afin de mieux promouvoir les productions francophones dans le domaine du management. C'est ainsi qu'ont été conçus les Vitrines de la RFG et le Festival de la RFG.

Les vitrines de la RFG sont des manifestations en format «court», destinées à promouvoir la parution d'un dossier spécial RFG. Elles sont organisées par les rédacteurs invités d'un dossier, en étroite concertation avec la rédaction de la revue. Après la vitrine inaugurale de septembre 2017 (Business Science Institute, Luxembourg), une seconde vitrine s'est tenue à Paris en octobre 2017 (Telecom ParisTech), avant d'enchaîner avec la vitrine RFG de l'IAE de Tours en mars 2018... un bon moyen de mieux faire rayonner encore les auteurs comme leurs institutions dans toutes leurs diversités. Grâce à l'ensemble du système de promotion de la revue sous forme de vidéos, de chroniques dans The Conversation France, de relais sur les médias sociaux, etc.

Mais la Revue française de gestion, c'est aussi, outre les articles varia et les dossiers spéciaux, un numéro double de fin d'année, choix éditorial assumé par le Conseil de rédaction afin de donner le « la », battre la mesure et ouvrir vers des sujets d'avenir. Cela méritait bien un tapis rouge et donc, un festival. En novembre 2017, l'IAE de Grenoble a accueilli le festival inaugural, organisé par Michel Albouy au nom du Conseil de rédaction sur le thème : «Finance, stratégie, gouvernance - 40 ans de la RFG ». Pour l'année 2018, le festival de la Revue française de gestion, 
organisé par Romain Laufer et Yvonne Muller-Lagarde, porte sur le thème « Le management face au judiciaire. Les défis de l'internormativité ».

Il est logique qu'en ayant impulsé autant de transformations aussi significatives, la Revue française de gestion ait reconquis sa couronne égarée. Elle a fièrement crevé le plafond du million de consultations d'articles via la plateforme Cairn, laquelle se joint désormais au rang des partenaires privilégiés des développements de la RFG. Les lecteurs découvriront, en annexes, les numéros, dossiers, articles, auteurs les plus consultés, « en institutions» et « hors institutions ». Et ô surprise, découvriront que la Revue française de gestion est aussi lue depuis l'étranger... C'est donc avec fierté, honneur et un profond sentiment de reconnaissance que la RFG remercie ses auteurs, évaluateurs, rédacteurs en chef invités et - surtout ! - lecteurs pour ce million de visites de nos productions ${ }^{1}$.

La seule «mauvaise nouvelle» de la période : après avoir perdu une place ces dernières années chez Google Scholar pour devenir deuxième revue francophone la plus citée, la Revue française de gestion a perdu brutalement début 2018 pas moins de... 17 places d'un coup et est reléguée désormais à la $19^{\mathrm{e}}$ place. Un déclassement brutal et d'autant plus surprenant que dans le même temps elle est passée de 400000 à plus d'un million de consultations sur Cairn.

Pour l'expliquer, on peut se risquer à formuler deux hypothèses : d'un côté, les « bad strategies » éditoriales pour le moins discutables au plan éthique de type «quote my journal in your research » finissent probablement par payer ; d'un autre côté, le lobbying de quelques éditeurs, associations, institutions pour solliciter des calculs d'impact sur trois ans et non sur l'histoire longue des revues rencontrent visiblement l'intérêt des data scientists et autres concepteurs d'algorithmes, avides de renouvellement du business.

Il est vrai que le monde académique, même s'il se plaît à s'en défendre, est aussi une industrie. Il serait donc étonnant que n'y fleurissent pas comme ailleurs quelques faux-semblants, croche-pieds et autres chausse-trappes. Alors, plutôt que de se désoler de ce nouveau coup du sort, on préfère se réjouir pour mieux continuer à faire tourner la roue. Parce que, fort heureusement, ce n'est pas demain la veille qu'une intelligence artificielle aura l'idée de créer un festival de Cannes pour faire exister le cinéma de Jacques Audiard à l'international et faire connaître au monde la valeur de nos exceptions culturelles francophones !

1. La liste des relecteurs 2017 se trouve à la fin de ce numéro. 


\section{LA RFG EN CHIFFRES}

\section{Les articles soumis}

\begin{tabular}{|l|l|c|}
\hline \multirow{2}{*}{2015} & Soumissions & Acceptés \\
\cline { 2 - 3 } & 238 & 34 \\
\hline \multirow{2}{*}{2016} & Soumissions & Acceptés \\
\cline { 2 - 3 } & 393 & 55 \\
\hline \multirow{2}{*}{2017} & Soumissions & Acceptés \\
\cline { 2 - 3 } & 330 & 25 \\
\hline
\end{tabular}

Source : la Revue française de gestion.

\section{Nombre de consulations d'articles en $\mathbf{2 0 1 7}$}

(43 articles, 5 numéros, mis en ligne)

$$
\text { En institutions }(\mathrm{N}=160 \text { 045) }
$$

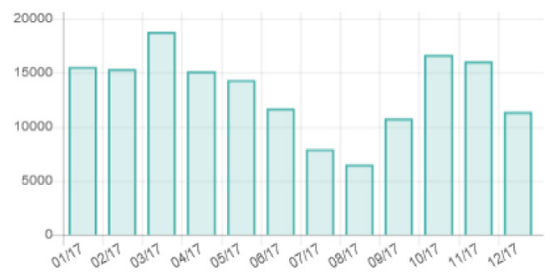

Source : statistiques.cairn.info, 2017.
Hors institutions $(\mathrm{N}=\mathbf{8 4 7} 057)$

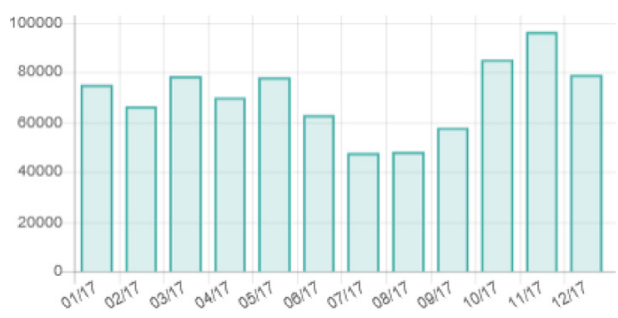

\section{Top 5 des institutions}

\begin{tabular}{|l|l|l|l|}
\hline \multicolumn{2}{|l|}{ Institutions francophones } & \multicolumn{2}{l|}{ Institutions non francophones } \\
\hline Aix-Marseille Université & 4898 & New York University & 220 \\
\hline Université Lyon 3 & 3846 & ESFAM & 138 \\
\hline Université Rennes 1 & 3761 & University of Bath & 122 \\
\hline Université Paris Dauphine & 3719 & University of Windsor & 118 \\
\hline Université Paris 1-Sorbonne & 3434 & West University of Timisoara & 87 \\
\hline
\end{tabular}

Source : statistiques.cairn.info, 2017. 


\section{Top 10 de articles (titre article, auteurs, dossier)}

\begin{tabular}{|c|c|c|c|}
\hline \multicolumn{2}{|l|}{ En institutions } & \multicolumn{2}{|l|}{ Hors institutions } \\
\hline $\begin{array}{l}\text { «L'émotion et la prise de } \\
\text { décision »- D. Van Hoorebeke, } \\
\left(2008, n^{\circ} 182\right)\end{array}$ & 1215 & $\begin{array}{l}\text { «L'émotion et la prise de } \\
\text { décision »-D. Van Hoorebeke, } \\
\left(2008, n^{\circ} 182\right)\end{array}$ & 13422 \\
\hline $\begin{array}{l}\text { «Marque employeur interne et } \\
\text { externe » - A. Charbonnier-Voirin } \\
\left(2015, n^{\circ} 246\right)\end{array}$ & 1126 & $\begin{array}{l}\text { «Management et travail social» } \\
\text { - B. Bouquet (2006, n' } 168-169)\end{array}$ & 11636 \\
\hline $\begin{array}{l}\text { « Expériences de consommation et } \\
\text { marketing expérientiel »- } \\
\text { A. Carù, B. Cova }\left(2006, n^{\circ} 162\right)\end{array}$ & 1084 & $\begin{array}{l}\text { « Expériences de consommation et } \\
\text { marketing expérientiel »- } \\
\text { A. Carù, B. Cova }\left(2006, n^{\circ} 162\right)\end{array}$ & 10886 \\
\hline $\begin{array}{l}\text { «La culture d'entreprise »- } \\
\text { É.Godelier }\left(2009, \mathrm{n}^{\circ} 192\right)\end{array}$ & 1028 & $\begin{array}{l}\text { «La relation entreprises-clients : } \\
\text { de la fidélité à la dépendance »- } \\
\text { V. Des Garets, É. Lamarque, } \\
\text { V. Plichon }\left(2003, n^{\circ} 144\right)\end{array}$ & 10832 \\
\hline $\begin{array}{l}\text { "Nike face à la controverse } \\
\text { éthique relative à ses sous- } \\
\text { traitants »- N. Gasmi, } \\
\text { G. Grolleau }\left(2005, n^{\circ} 157\right)\end{array}$ & 916 & $\begin{array}{l}\text { «La culture d'entreprise »- } \\
\text { É. Godelier }\left(2009, n^{\circ} 192\right)\end{array}$ & 10282 \\
\hline $\begin{array}{l}\text { «Expériences de consommation et } \\
\text { marketing expérientiel »- } \\
\text { A. Carù, B. Cova }\left(2015, \mathrm{n}^{\circ} \text { 253) }\right.\end{array}$ & 889 & $\begin{array}{l}\text { «Méthode d'analyse des } \\
\text { discours »-A. Seignour (2011, } \\
\left.\mathrm{n}^{\circ} 211\right)\end{array}$ & 9077 \\
\hline $\begin{array}{l}\text { «Management de l'innovation et } \\
\text { nouvelle ère numérique »- } \\
\text { P.-J. Barlatier }\left(2016, \mathrm{n}^{\mathrm{o}} 254\right)\end{array}$ & 880 & $\begin{array}{l}\text { «L'approche relationnelle dans } \\
\text { les banques »- V. Des Garets, } \\
\text { M. Paquerot, I. Sueur (2009, n } 191)\end{array}$ & 8800 \\
\hline $\begin{array}{l}\text { «Management et travail social » } \\
- \text { B. Bouquet (2006, n 168-169) }\end{array}$ & 800 & $\begin{array}{l}\text { «Théorie financière et stratégie } \\
\text { financière »-G. Charreaux } \\
\left(2006, \mathrm{n}^{\circ} 160\right)\end{array}$ & 8384 \\
\hline $\begin{array}{l}\text { «La conduite du changement »- } \\
\text { É. Minvielle, } \\
\text { A.-P. Contandriopoulos }(2004, \\
\left.n^{\circ} 150\right)\end{array}$ & 759 & $\begin{array}{l}\text { «Proposition d'un outil } \\
\text { d'évaluation de l'autonomie dans } \\
\text { le travail »-C. Everaere ( } 2007 \text {, } \\
\left.n^{\circ} 180\right)\end{array}$ & 8300 \\
\hline $\begin{array}{l}\text { « Méthode d'analyse des } \\
\text { discours » - A. Seignour (2011, } \\
\left.\mathrm{n}^{\circ} 211\right)\end{array}$ & 732 & $\begin{array}{l}\text { «Nike face à la controverse } \\
\text { éthique relative à ses sous- } \\
\text { traitants »- N. Gasmi, } \\
\text { G. Grolleau }\left(2005, \mathrm{n}^{\circ} 157\right)\end{array}$ & 8064 \\
\hline
\end{tabular}

Source : statistiques.cairn.info, 2017. 


\section{Tops 10 des auteurs}

\begin{tabular}{|l|l|l|l|}
\hline \multicolumn{2}{|l|}{ En institutions } & Hors institutions \\
\hline Alain-Charles Martinet & 2116 & Éric Lamarque & 20524 \\
\hline Bernard Cova & 2099 & Véronique Des Garets & 19632 \\
\hline Antonella Carù & 1973 & Gérard Charreaux & 14076 \\
\hline Gilles Paché & 1942 & Delphine Van Hoorebeke & 13422 \\
\hline Frédéric Le Roy & 1937 & Véronique Plichon & 12010 \\
\hline Christophe Everaere & 1516 & Gilles Paché & 11957 \\
\hline Emmanuelle Reynaud & 1449 & Brigitte Bouquet & 11636 \\
\hline Véronique Des Garets & 1389 & Frédéric Le Roy & 11123 \\
\hline Michel Albouy & 1367 & Éric Godelier & 10963 \\
\hline Caroline Mothe & 1352 & Bernard Cova & 10956 \\
\hline
\end{tabular}

Source : statistiques.cairn.info, 2017.

\section{Top 10 des pays}

\begin{tabular}{|l|l|l|l|}
\hline \multicolumn{2}{|l|}{ En institutions } & Hors institutions \\
\hline France & 121980 & France & 433763 \\
\hline Canada & 13367 & Maroc & 83607 \\
\hline Belgique & 7657 & Tunisie & 36719 \\
\hline Maroc & 4139 & États-Unis avertissement & 36118 \\
\hline Suisse & 3272 & Algérie & 31875 \\
\hline Tunisie & 2841 & Canada & 25838 \\
\hline Algérie & 1991 & Belgique & 22997 \\
\hline Liban & 588 & Cameroun & 22451 \\
\hline Sénégal & 574 & Côte d'Ivoire & 16211 \\
\hline Cameroun & 525 & Royaume-Uni & 14264 \\
\hline
\end{tabular}

Source : statistiques.cairn.info, 2017. 